\title{
WEAKLY NONLINEAR BOUNDARY-VALUE PROBLEMS FOR OPERATOR EQUATIONS WITH PULSE INFLUENCE
}

\author{
A. M. Samoilenko, A. A. Boichuk, and V. F. Zhuravlev
}

UDC 517.9

\begin{abstract}
We consider the problem of finding conditions of solvability and algorithms for construction of solutions of weakly nonlinear boundary-value problems for operator equations (with the Noetherian linear part) with pulse influence at fixed times. The method of investigation is based on passing by methods of the Lyapunov-Schmidt type from a pulse boundary-value problem to an equivalent operator system that can be solved by iteration procedures based on the fixed-point principle.
\end{abstract}

The study of the qualitative theory of differential systems with pulse influence started in $[1,2]$ has been further developed and extended in a great number of papers. Thus, an approach to the study of pulse periodic boundaryvalue problems for ordinary differential systems proposed in [1] was successfully developed and applied in the case of weakly nonlinear pulse boundary-value problems (with Noetherian linear part) for ordinary differential systems [3] and for linear boundary-value problems for differential systems with concentrated lag [4]. From the point of view of operator theory, one of the essential peculiarities of these problems is the everywhere solvability [5] of the Cauchy problem for initial differential systems. However, there exists a fairly wide class of boundary-value problems for functional differential equations [6] for which the initial operator equation is not everywhere solvable. Precisely this class of problems is considered in the present paper; our purpose is to obtain criteria for the solvability and formulas for the presentation of solutions of linear boundary-value problems for Noetherian operator equations with pulse influence at fixed times. We find conditions of solvability for weakly nonlinear (with Noetherian linear part) pulse boundary-value problems and construct a convergent iterative algorithm for finding solutions. For this purpose, we use criteria of solvability and formulas obtained in [3] for constructing solutions of linear Noetherian operator equations in Banach and Hilbert spaces. By methods of the Lyapunov-Schmidt type, we pass from a pulse boundary-value problem to an operator system and then apply the convergent iterative procedures based on the fixed-point principle [7, 8].

Denote an interval $-\infty<a \leq t \leq b<+\infty$ by $I_{[a, b]}$, an interval $0 \leq \varepsilon \leq \varepsilon_{0}$ by $I_{\left[0, \varepsilon_{0}\right]}$, a space of $n$ dimensional vectors $x=\operatorname{col}\left[x_{1}, x_{2}, \ldots, x_{n}\right]$ with the norm $\|x\|=\max _{1 \leq i \leq n}\left|x_{i}\right|$ by $R^{n}$, a space of $(n \times n)$ dimensional matrices with a norm consistent with a norm in $R^{n}$ by $R^{n \times n}$, and the Heaviside function by $\chi_{\tau}(t)$, i.e., $\chi_{\tau}(t)=0$ for $t \leq \tau$ and $\chi_{\tau}(t)=1$ for $t>\tau$. By analogy with [6, p. 123], we introduce the following spaces of functions $x:[a, b] \rightarrow R^{n}: L^{n}$ is a space of summable functions with norm

$$
\|x\|_{L^{n}}=\int_{a}^{b}\|x(s)\| d s
$$

$D^{n}$ is a space of absolutely continuous functions with norm $\|x\|_{D^{n}}=\|x(a)\|+\|\dot{x}\|_{L^{n}} ; C$ is a space of continuous functions with norm $\|x\|_{C}=\max _{s \in[a, b]}\|x(s)\| ; B V$ is a space of functions of bounded variation with norm $\|x\|_{B V}=\|x(a)\|+\operatorname{var}_{a}^{b} x ; S P$ is a subspace of the space $B V$ consisting of functions of jumps:

$$
S P=\left\{y \in B V \mid y=\sum_{i=1}^{n} a_{i} \chi_{\tau_{i}}, a_{i} \in R^{n}, \tau_{i} \in[a, b), i=1, \ldots, n, \tau_{i} \neq \tau_{j}, \text { if } i \neq j\right\}
$$

Institute of Mathematics, Ukrainian Academy of Sciences, Kiev. Translated from Ukrainskii Matematicheskii Zhurnal, Vol. 49, No. 2, pp. 272-288, February, 1997. Original article submitted October 14, 1996. 
with a norm induced by the norm of $B V$; and $D^{n} S$ is a subspace of the space $B V$ consisting of functions that can be represented as the sum of an absolutely continuous function and a function of jumps:

$$
D^{n} S=\left\{z \in B V \mid \exists x \in D^{n}, \exists y \in S P: z=x+y\right\}, \quad\|z\|_{D^{n} S}=\|x\|_{D^{n}}+\|y\|_{S P} .
$$

\section{Statement of the Problem}

Consider a weakly nonlinear operator equation

$$
(L z)(t)=f(t)+\varepsilon Z(z, t, \varepsilon)
$$

where $L: D^{n} \rightarrow L^{n}$ is a linear bounded Noetherian operator [5, p. 38] (ind $L=\operatorname{dim} \operatorname{ker} L-\operatorname{dim} \operatorname{ker} L^{*}=s-k$ ), $L^{*}$ is an operator adjoint to the operator $L, Z: D^{n} \times I_{[a, b]} \times I_{\left[0, \varepsilon_{0}\right]} \rightarrow L^{n}$ is a nonlinear operator, and $\varepsilon$ is a small nonnegative parameter.

Let $\tau_{1}, \tau_{2}, \ldots, \tau_{p}$ be a fixed strictly ordered system of points from the interval $[a, b]$. Assume that solutions of Eq. (1) at times $\tau_{i}, i=1,2, \ldots, p$, have jumps defined by the equalities

$$
\left.\Delta z\right|_{i=\tau_{i}}-S_{i} z\left(\tau_{i}-, \varepsilon\right)=a_{i}+\varepsilon J_{i}\left(z\left(\tau_{i}-, \varepsilon\right), \varepsilon\right),
$$

where $\left.\Delta z\right|_{t=\tau_{i}}=z\left(\tau_{i}+, \varepsilon\right)-z\left(\tau_{i}-, \varepsilon\right), S_{i} \in R^{n \times n}$ are such that $\operatorname{det}\left(E+S_{i}\right) \neq 0, J_{i}: D^{n} \times I_{\left[0, \varepsilon_{0}\right]} \rightarrow R^{n}$ is a nonlinear vector functional, and $a_{i} \in R^{n}$.

Assume that solutions of system (1), (2) satisfy the conditions

$$
l z(\cdot, \varepsilon)=\alpha+\varepsilon J(z(\cdot, \varepsilon), \varepsilon)
$$

where $l: D^{n} \times I_{\left[0, \varepsilon_{0}\right]} \rightarrow R^{m}$ and $J: D^{n} \times I_{\left[0, \varepsilon_{0}\right]} \rightarrow R^{m}$ are $m$-dimensional linear and nonlinear bounded vector functionals, respectively.

\section{Linear Boundary-Value Problems}

Before studying the weakly nonlinear pulse boundary-value problem (1)-(3), we consider the problem of finding a criterion of solvability and formulas for the presentation of solutions of the generating linear boundaryvalue problem obtained from (1) -(3) if $\varepsilon=0$, namely,

$$
\begin{gathered}
(L z)(t)=f(t), \quad t \in[a, b], \\
\left.\Delta z\right|_{t=\tau_{i}}-S_{i} z\left(\tau_{i}-, 0\right)=a_{i} \\
l z(\cdot, 0)=\alpha .
\end{gathered}
$$

A function $z(t) \in D^{n} S$ satisfying Eq. (4) for almost all $t \in[a, b]$ and conditions (5) and (6) is called a solution of the linear pulse boundary-value problem (4)-(6).

It is known [3, p. 53] that the linear Noetherian operator equation (4) is solvable for those and only those $f(t) \in L^{n}$ which satisfy the condition $\left(P_{Y} f\right)(t)=0$, and its solution has the form 


$$
z\left(t, c_{s}\right)=X(t) c_{s}+\left(L^{-} f\right)(t)
$$

where $X(t)$ is an $(n \times s)$-dimensional matrix formed by basis vectors of a null space $N(L)$ of the operator $L$; $P_{Y}: L^{n} \rightarrow Y$ is a projector on a subspace $Y \in L^{n}$ isomorphic to a null space $N\left(L^{*}\right)$ of the operator $L^{*}$; and $L^{-}$ is a generalized inverse operator of the Noetherian operator $L$.

For the pulse Noetherian operator equation (4), (5), the following statement is true:

Theorem 1. The Noetherian operator equation with pulse influence (4), (5) is solvable for any $a_{i} \in R^{n}$, $i=1,2, \ldots, p$, and for those and only those $f(t) \in L^{n}$ which satisfy the condition

$$
\left(P_{Y} f\right)(t)=0
$$

The equation considered has a general solution of the form

$$
z(t, c)=\bar{X}(t) c+\left(\tilde{L}^{-}\left[\begin{array}{l}
f \\
a_{i}
\end{array}\right]\right)(t), \quad c \in R^{s},
$$

where

$$
\left.\left.\bar{X}(t)=X(t) \sum_{i=1}^{k} \prod_{v=i}^{k-1} \bar{S}_{v} P_{i-1}, \quad t \in\right] \tau_{k-1}, \tau_{k}\right]
$$

is an $(n \times s)$-dimensional matrix formed by basis vectors of a null space of the pulse Noetherian operator $\Lambda=\operatorname{col}\left[L, \Delta-S_{i}\right]$

$$
\left.\left.\left(\tilde{L}^{-}\left[\begin{array}{l}
f \\
a_{i}
\end{array}\right]\right)(t)=\left(L^{-} f\right)(t)+\sum_{i=1}^{k-1}\left(L_{i}^{-} f\right)(t)+\sum_{i=1}^{k-1} \tilde{X}_{i}(t) a_{i}, \quad t \in\right] \tau_{k-1}, \tau_{k}\right]
$$

is a generalized inverse operator of the operator of pulse equation (4), (5). Here,

$$
\begin{gathered}
\left(\tilde{L}_{i}^{-} f\right)(t)=X(t) \prod_{v=i+1}^{k-1} \bar{S}_{v}\left(L_{i}^{-} f\right)\left(\tau_{i}\right), \\
\tilde{X}_{i}(t)=X(t) \prod_{v=i+1}^{k-1} \bar{S}_{v} X^{+}\left(\tau_{i}\right), \quad P_{0}=E_{s}, \quad \prod_{v=k+1}^{k} \bar{S}_{v}=E_{s}, \\
\bar{S}_{i}=X^{+}\left(\tau_{i}\right)\left(E+S_{i}\right) X\left(\tau_{i}\right) ; \quad\left(L_{i}^{-} f\right)\left(\tau_{i}\right)=X^{+}\left(\tau_{i}\right) S_{i}\left(L^{-} f\right)\left(\tau_{i}\right),
\end{gathered}
$$

where $X^{+}\left(\tau_{i}\right)$ is an $(s \times n)$-dimensional unique pseudoinverse matrix of an $(n \times s)$-dimensional constant matrix $X\left(\tau_{i}\right) ; P_{i}: R^{s} \rightarrow N\left(X\left(\tau_{i}\right)\right)$ is an $(s \times s)$-dimensional orthoprojector matrix of the Euclidean space $R^{s}$ on a null space $N\left(X\left(\tau_{i}\right)\right)$ of the matrix $X\left(\tau_{i}\right)$.

Proof. Assume that condition (8) of solvability of Noetherian operator equation (4) holds. Then a general solution of this equation on the intervals $\left[a, \tau_{1}\right]$ and $\left.] \tau_{1}, \tau_{2}\right]$ has the form 


$$
\begin{gathered}
z_{1}(t)=X(t) c+\left(L^{-} f\right)(t), \quad t \in\left[a, \tau_{1}\right], \\
\left.\left.z_{2}(t)=X(t) c_{2}+\left(L^{-} f\right)(t), \quad t \in\right] \tau_{1}, \tau_{2}\right] .
\end{gathered}
$$

By pulse conditions (5), we obtain

$$
X\left(\tau_{1}+\right) c_{2}+\left(L^{-} f\right)\left(\tau_{1}+\right)=\left(E+S_{1}\right)\left\{X\left(\tau_{1}-\right) c+\left(L^{-} f\right)\left(\tau_{1}-\right)\right\}+a_{1}
$$

For simplicity, without loss of generality, we assume everywhere below that

$$
X\left(\tau_{i}-\right)=X\left(\tau_{i}+\right), \quad\left(L^{-} f\right)\left(\tau_{i}^{-}\right)=\left(L^{-} f\right)\left(\tau_{i}+\right)
$$

Denote by $P_{1}$ an $(s \times s)$-dimensional orthoprojector matrix projecting the Euclidean space $R^{s}$ on the null space $N\left(X\left(\tau_{1}\right)\right)$ of a constant $(s \times n)$-dimensional matrix $X\left(\tau_{1}\right), P_{1}: R^{s} \rightarrow N\left(X\left(\tau_{1}\right)\right)$. Let $P_{1}^{(*)}$ denote an $(n \times n)$-dimensional orthoprojector matrix projecting the Euclidean space $R^{n}$ on the null space $N\left(X^{*}\left(\tau_{1}\right)\right)$ of an $(n \times s)$-dimensional matrix $X^{*}\left(\tau_{1}\right)$ adjoint to the matrix $X\left(\tau_{1}\right), P_{1}^{(*)}: R^{n} \rightarrow N\left(X^{*}\left(\tau_{1}\right)\right)$.

It is known that system (11) is solvable with respect to $c \in R^{s}$ if and only if

$$
P_{1}^{(*)}\left\{\left(E+S_{1}\right) X\left(\tau_{1}\right) c+S_{1}\left(L^{-} f\right)\left(\tau_{1}\right)+a_{1}\right\}=0
$$

Since the $(n \times s)$-dimensional matrix $X(t)$ is formed by a complete system of $s$ linearly independent basis vectors $\left\{f_{i}\right\}_{i=1}^{s}$, we have that the constant matrix $X\left(\tau_{1}\right)$ has the complete rank $\left[\operatorname{rank} X\left(\tau_{1}\right)=n, n \leq s\right]$. Therefore, $P_{1}^{(*)} \equiv 0$ and, consequently, condition (12) always holds. In addition, system (11) has an $\left(r_{0}=s-n\right)$-parametric family of solutions

$$
c_{2}=P_{1} c+X^{+}\left(\tau_{1}\right)\left(E+S_{1}\right) X\left(\tau_{1}\right) c+X^{+}\left(\tau_{1}\right) S_{1}\left(L^{-} f\right)\left(\tau_{1}\right)+X^{+}\left(\tau_{1}\right) a_{1}
$$

where $X^{+}\left(\tau_{1}\right)$ is a unique $(s \times n)$-dimensional pseudoinverse matrix of the matrix $X\left(\tau_{1}\right)$, which can be constructed by the relation [3, p. 91]

$$
X^{+}\left(\tau_{1}\right)=X^{*}\left(\tau_{1}\right)\left[X\left(\tau_{1}\right) X^{*}\left(\tau_{1}\right)\right]^{-1}
$$

with regard for the facts that $P_{N\left(X^{*}\left(\tau_{1}\right)\right)}=0$ and $P_{1} c \in N\left(X\left(\tau_{1}\right)\right)$, where $c \in R^{s}$ is an arbitrary $s$-dimensional vector.

By substituting the obtained value of $c_{2}$ into relation (10), we arrive at the expression for the general solution $z_{2}(t)$ of the pulse operator equation (4), (5) on the interval $\left.] \tau_{1}, \tau_{2}\right]$ :

$$
z_{2}(t)=X(t)\left[P_{1}+X^{+}\left(\tau_{1}\right)\left(E+S_{1}\right) X\left(\tau_{1}\right)\right] c+\left(L^{-} f\right)(t)+X(t) X^{+}\left(\tau_{1}\right) S_{1}\left(L^{-} f\right)\left(\tau_{1}\right)+X(t) X^{+}\left(\tau_{1}\right) a_{1} .
$$

If we substitute the notation

$$
\bar{S}_{1}=X^{+}\left(\tau_{1}\right)\left(E+S_{1}\right) X\left(\tau_{1}\right), \quad\left(L_{1}^{-} f\right)\left(\tau_{1}\right)=X^{+}\left(\tau_{1}\right) S_{1}\left(L^{-} f\right)\left(\tau_{1}\right)
$$

into this expression, we rewrite it in the form 


$$
z_{2}(t)=X(t)\left[\bar{S}_{1}+P_{1}\right] c+\left(L^{-} f\right)(t)+X(t)\left(L_{1}^{-} f\right)\left(\tau_{1}\right)+X(t) X^{+}\left(\tau_{1}\right) a_{1}
$$

On the interval $] \tau_{2}, \tau_{3}$ ], a solution of the Noetherian operator equation (4) has the form

$$
\left.\left.z_{3}(t)=X(t) c_{3}+\left(L^{-} f\right)(t), \quad t \in\right] \tau_{2}, \tau_{3}\right]
$$

In view of pulse conditions (5), we have

$$
\begin{aligned}
X\left(\tau_{2}+\right) c_{3}+\left(L^{-} f\right)\left(\tau_{2}+\right)=\left(E+S_{2}\right)\left\{X ( \tau _ { 2 } ^ { - } ) \left[\bar{S}_{1}\right.\right. & \left.+P_{1}\right] c+\left(L^{-} f\right)\left(\tau_{2}-\right) \\
& \left.+X\left(\tau_{2}-\right)\left(L_{1}^{-} f\right)\left(\tau_{1}\right)+X\left(\tau_{2}-\right) X^{+}\left(\tau_{1}\right) a_{1}\right\}+a_{2} .
\end{aligned}
$$

As before, we denote by $P_{2}$ an $(s \times s)$-dimensional orthoprojector matrix, $P_{2}: R^{s} \rightarrow N\left(X\left(\tau_{2}\right)\right)$, and by $P_{2}^{(*)}$ an $(n \times n)$-dimensional orthoprojector matrix, $P_{2}^{(*)}: R^{n} \rightarrow N\left(X^{*}\left(\tau_{2}\right)\right)$.

Since the constant $(n \times s)$-dimensional matrix $X\left(\tau_{2}\right)$ has complete rank, we have that $P_{2}^{(*)} \equiv 0$. Moreover, Eq. (14) is always solvable with respect to $c_{3} \in R^{s}$ and has an $\left(r_{0}=s-n\right)$-parametric family of solutions

$$
\begin{aligned}
c_{3}=P_{2} c & +X^{+}\left(\tau_{2}\right)\left(E+S_{2}\right) X\left(\tau_{2}\right)\left[\bar{S}_{1}+P_{1}\right] c+X^{+}\left(\tau_{2}\right) S_{2}\left(L^{-} f\right)\left(\tau_{2}\right) \\
& +X^{+}\left(\tau_{2}\right)\left(E+S_{2}\right) X\left(\tau_{2}\right)\left(L_{1}^{-} f\right)\left(\tau_{1}\right)+X^{+}\left(\tau_{2}\right)\left(E+S_{2}\right) X\left(\tau_{2}\right) X^{+}\left(\tau_{1}\right) a_{1}+X^{+}\left(\tau_{2}\right) a_{2} .
\end{aligned}
$$

If we introduce the notation

$$
\bar{S}_{2}=X^{+}\left(\tau_{2}\right)\left(E+S_{2}\right) X\left(\tau_{2}\right), \quad\left(L_{2}^{-} f\right)\left(\tau_{2}\right)=X^{+}\left(\tau_{2}\right) S_{2}\left(L^{-} f\right)\left(\tau_{2}\right)
$$

and substitute the obtained value of $c_{3}$ into Eq. (13), we arrive at the expression for the solution $z_{3}(t)$ on the interval $\left.] \tau_{2}, \tau_{3}\right]$ :

$$
\begin{aligned}
z_{3}(t)=X(t)\left[\bar{S}_{2} \bar{S}_{1}+\bar{S}_{2} P_{1}+P_{2}\right] c & +\left(L^{-} f\right)(t) \\
& +X(t)\left[\bar{S}_{2}\left(L_{1}^{-} f\right)\left(\tau_{1}\right)+\left(L_{2}^{-} f\right)\left(\tau_{2}\right)\right]+X(t)\left[\bar{S}_{2} X^{+}\left(\tau_{1}\right) a_{1}+X^{+}\left(\tau_{2}\right) a_{2}\right]
\end{aligned}
$$

By applying a similar reasoning to the solution $z_{4}(t)$ on the interval $] \tau_{3}, \tau_{4}$, we obtain

$$
\begin{aligned}
z_{4}(t)=X(t)\left[\bar{S}_{3} \bar{S}_{2} \bar{S}_{1}+\bar{S}_{3} \bar{S}_{2} P_{1}+\bar{S}_{3} P_{2}+P_{3}\right] c & +\left(L^{-} f\right)(t) \\
& +X(t)\left[\bar{S}_{3} \bar{S}_{2}\left(L_{1}^{-} f\right)\left(\tau_{1}\right)+\bar{S}_{3}\left(L_{2}^{-} f\right)\left(\tau_{2}\right)+\left(L_{3}^{-} f\right)\left(\tau_{3}\right)\right] \\
& +X(t)\left[\bar{S}_{3} \bar{S}_{2} X^{+}\left(\tau_{1}\right) a_{1}+\bar{S}_{3} X^{+}\left(\tau_{2}\right) a_{2}+X^{+}\left(\tau_{3}\right) a_{3}\right] .
\end{aligned}
$$

By continuing this procedure, we obtain the statement of the theorem for the Noetherian operator equation with pulse influence.

Remark 1. If Eq. (4) has a solution for any $f(t) \in L^{n}$, then condition (8) in Theorem 1 always holds. In this 
case, pulse operator equation (4), (5) is everywhere solvable, and this assertion holds, for example, for pulse ordinary differential systems [3, p. 235] and for pulse systems with lag [4]. Moreover, in the case of ordinary differential systems, relation (9) is considerably simplified [1].

Further, let us discuss the problems of solvability and representation of a general solution of the generating boundary-value problem (4) - (6). In order that solution (9) of pulse Noetherian operator equation (4), (5) satisfy boundary conditions (6), it is necessary and sufficient that $l z(\cdot, c)=\alpha$. The last relation yields the following algebraic system for finding a vector constant $c \in R^{s}$ :

$$
Q c=\alpha-l\left(\tilde{L}^{-}\left[\begin{array}{l}
f \\
a_{i}
\end{array}\right]\right)(\cdot)
$$

[here, $Q=l X(\cdot)$ is an $(m \times s)$-dimensional constant matrix]. Assume that $\operatorname{rank} Q=r_{1}$.

Equation (15) is solvable with respect to $c \in R^{s}$ if and only if

$$
P_{N\left(Q^{*}\right)_{d}}\left\{\alpha-l\left(\tilde{L}^{-}\left[\begin{array}{l}
f \\
a_{i}
\end{array}\right]\right)(\cdot)\right\}=0, \quad d=m-r_{l}
$$

and, in this case, it has an $\left(r=n-r_{1}\right)$-parameter family of solutions

$$
c=P_{N(Q)_{r}} c_{r}+Q^{+} \alpha-Q^{+} l\left(\tilde{L}^{-}\left[\begin{array}{l}
f \\
a_{i}
\end{array}\right]\right)(\cdot) .
$$

Here, $Q^{+}$is a unique $(s \times m)$-dimensional matrix pseudoinverse to $Q, P_{N(Q)}$ is an $(s \times r)$-dimensional matrix whose columns form a complete system of $r$ linearly independent columns of the orthoprojector matrix $P_{N(Q)}: R^{s} \rightarrow N(Q)$, and $P_{N\left(Q^{*}\right)_{d}}$ is a $(d \times m)$-dimensional matrix whose rows form a complete system of $d$ linearly independent rows of the orthoprojector matrix $P_{N\left(Q^{*}\right)}[3$, p.91].

By substituting solution (16) into (9), we obtain a general solution of the linear boundary-value problem for the pulse Noetherian operator equation of the form

$$
\begin{aligned}
z\left(t, c_{r}\right)=\bar{X}(t) P_{N(Q)} c_{r}+\bar{X}(t) Q^{+} \alpha & +\left(L^{-} f\right)(t) \\
& -\bar{X}(t) Q^{+} l\left(L^{-} f\right)(\cdot)+\sum_{i=1}^{k-1}\left(\tilde{L}_{i}^{-} f\right)(t)-\sum_{i=1}^{p} \bar{X}(t) Q^{+} l\left(\tilde{L}_{i}^{-} f\right)(\cdot) \\
& \left.\left.+\sum_{i=1}^{k-1} \tilde{X}_{i}(t) a_{i}-\sum_{i=1}^{p} \bar{X}(t) Q^{+} l \tilde{X}_{i}(\cdot) a_{i}, \quad t \in\right] \tau_{k-1}, \tau_{k}\right] .
\end{aligned}
$$

Thus, the following theorem is true:

Theorem 2. The linear pulse boundary-value problem (4)-(6) for the Noetherian operator equation is solvable for those and only those $f(t) \in L^{n}, \alpha \in R^{m}$, and $a_{i} \in R^{n}$ which satisfy the conditions

$$
\left(P_{Y} f\right)(t)=0,
$$




$$
P_{N\left(Q^{*}\right)}\left\{\alpha-l\left(\tilde{L}^{-}\left[\begin{array}{l}
f \\
a_{i}
\end{array}\right]\right)(\cdot)\right\}=0 .
$$

A general solution of this system has the form

$$
z\left(t, c_{r}\right)=\bar{X}(t) c_{r}+\left(\tilde{G}\left[\begin{array}{l}
f \\
a_{i}
\end{array}\right]\right)(t)+\bar{X}(t) Q^{+} \alpha
$$

where $\bar{X}_{r}(t)=\bar{X}(t) P_{N(Q)}$ is an $(n \times r)$-dimensional matrix formed by the basis vectors of null space of the operator $\Lambda_{1}=\operatorname{col}[\Lambda, l]$;

$$
\begin{aligned}
\left(\tilde{G}\left[\begin{array}{l}
f \\
\\
a_{i}
\end{array}\right]\right)(t)=\left(L^{-} f\right)(t)-\bar{X}(t) Q^{+} l\left(L^{-} f\right)(\cdot) & +\sum_{i=1}^{k-1}\left(\tilde{L}_{i}^{-} f\right)(t) \\
& -\sum_{i=1}^{p} \bar{X}(t) Q^{+} l\left(\tilde{L}_{i}^{-} f\right)(\cdot) \\
& \left.\left.+\sum_{i=1}^{k-1} \tilde{X}_{i}(t) a_{i}-\sum_{i=1}^{p} \bar{X}(t) Q^{+} l \tilde{X}_{i}(\cdot) a_{i}, \quad t \in\right] \tau_{k-1}, \tau_{k}\right]
\end{aligned}
$$

is the generalized Green operator for a semihomogeneous $(\alpha=0)$ pulse boundary-value problem corresponding to problem (4)-(6).

Remark 2. If linear operator equation (4) is everywhere solvable [5, p. 8] as occurs, for example, for pulse differential systems with or without lag, then condition (17) in Theorem 2 is absent, and this theorem reduces to the known theorems $[3$, p. 238; 4] for corresponding classes of systems.

\section{Weakly Nonlinear Boundary-Value Problems}

Consider a weakly nonlinear boundary-value problem

$$
\begin{gathered}
(L z)(t)=f(t)+\varepsilon Z(z, t, \varepsilon), \\
\left.\Delta z\right|_{t=\tau_{i}}-S_{i} z\left(\tau_{i}-, \varepsilon\right)=a_{i}+\varepsilon J_{i}\left(z\left(\tau_{i}-, \varepsilon\right), \varepsilon\right), \\
l z(\cdot, \varepsilon)=\alpha+\varepsilon J(z(\cdot, \varepsilon), \varepsilon) .
\end{gathered}
$$

Assume that:

(a $\left.a_{1}\right) L: D^{n} \rightarrow L^{n}$ is a linear bounded Noetherian operator;

(a) $Z: D^{n} \times I_{[a, b]} \times I_{\left[0, \varepsilon_{0}\right]} \rightarrow L^{n}$ is a nonlinear operator continuous in $z$, Fréchet continuously differentiable with respect to $z$ in a neighborhood of a generating solution, continuous in $\varepsilon$, and

$$
Z(0, t, 0)=0, \quad \partial Z(0, t, 0) \backslash \partial z=0
$$

(a) $J_{i}: D^{n} \times I_{\left[0, \varepsilon_{0}\right]} \rightarrow R^{m}$ and $J: D^{n} \times I_{\left[0, \varepsilon_{0}\right]} \rightarrow R^{m}$ are nonlinear $n$ - and $m$-dimensional functionals in $z$, respectively, which are Fréchet continuously differentiable in a neighborhood of solutions of a 
generating boundary-value problem, continuous in $\varepsilon$, and

$$
J_{i}(0,0)=0, \quad \partial J_{i z}(0,0) \backslash \partial z=0 ; \quad J(0,0)=0, \quad \partial J_{z}(0,0) \backslash \partial z=0 ;
$$

(a $\left.a_{4}\right) l: D^{n} C \rightarrow R^{m}$ is a linear bounded $m$-dimensional vector functional;

(a $\left.\mathrm{a}_{5}\right) f(t) \in L^{n}, \alpha \in R^{m}, a_{i} \in R^{n}, i=1,2, \ldots, p$.

The function $z(t, \varepsilon)$ continuous in $\varepsilon$, satisfying Eq. (1) for almost all $t \in[a, b]$, and satisfying conditions (2), (3) is called a solution of the pulse boundary-value problem (21). In what follows, we denote by $D^{n} S C$ a space of solutions of this sort.

Consider the problem of finding existence conditions and the algorithm for construction of solutions $z(t, \varepsilon)$, which belong to the space $D^{n} S C$ and, for $\varepsilon=0$, are transformed into one of the generating solutions $z_{0}\left(t, c_{r}\right)$ (19) of the generating boundary-value problem (4)-(6). We denote the space of solutions of this sort by $D^{n} S C_{0}$.

Let us solve the stated problem in the following way: By using the generalized Green operator (20) constructed above, we reduce the initial boundary-value problem (21) to an equivalent operator system and then apply to this system the method of simple iterations under the assumption that the conditions of Theorem 1 hold, i.e., that $f(t) \in L^{n}, \alpha \in R^{m}, a_{i} \in R^{n}, i=1,2, \ldots, p$, satisfy conditions (17), (18), and the generating boundary-value problem (4)-(6) has an $r$-parametric family of solutions (19).

The following theorem establishes necessary conditions of the existence of solutions $z(t, \varepsilon) \in D^{n} S C_{0}$ of the weakly nonlinear pulse boundary-value problem (21):

Theorem 3. Let the weakly nonlinear boundary-value problem (21) have a solution $z(t, \varepsilon) \in D^{n} S C$ which, for $\varepsilon=0$, reduces to one of generating solutions of the linear pulse boundary-value problem (4)-(6) [this problem is generating for (21)] with a constant $c_{r}=c_{0} \in R^{r}$. Then the vector $c_{0}$ satisfies the system of equations

$$
\begin{gathered}
\left(P_{Y} Z\left(z_{0}\left(\cdot, c_{r}\right), \cdot, 0\right)\right)(t)=0 \\
P_{N\left(Q^{*}\right)_{d}}\left\{J\left(z_{0}\left(\cdot, c_{r}\right), 0\right)-l\left(\tilde{L}^{-}\left[\begin{array}{l}
Z\left(z_{0}\left(s, c_{r}\right), s, 0\right) \\
J_{i}\left(z_{0}\left(\tau_{i}-, c_{r}\right), 0\right)
\end{array}\right]\right)(\cdot)\right\}=0
\end{gathered}
$$

Proof. Assume that the conditions of the theorem are satisfied. Hence, for all $0 \leq \varepsilon \leq \varepsilon_{0}$ and for almost all $t \in[a, b]$, the identities

$$
\begin{gathered}
(L z)(t) \equiv f(t)+\varepsilon Z(z, t, \varepsilon), \\
\left.\Delta z\right|_{t=\tau_{i}}-S_{i} z\left(\tau_{i}-, \varepsilon\right) \equiv a_{i}+\varepsilon J_{i}\left(z\left(\tau_{i}-, \varepsilon\right), \varepsilon\right), \\
l z(\cdot, \varepsilon) \equiv \alpha+\varepsilon J(z(\cdot, \varepsilon), \varepsilon)
\end{gathered}
$$

hold. It follows from Theorem 2 that the operators $Z, J$, and $J_{i}$ satisfy, for $0 \leq \varepsilon \leq \varepsilon_{0}$, conditions of the form (17), (18), i.e.,

$$
\left(P_{Y} Z\left(z_{0}\left(\cdot, c_{r}\right), \cdot, \varepsilon\right)\right)(t)=0
$$




$$
P_{N\left(Q^{*}\right)_{d}}\left\{J\left(z_{0}\left(\cdot, c_{r}\right), \varepsilon\right)-l\left(\tilde{L}^{-}\left[\begin{array}{l}
Z\left(z_{0}\left(s, c_{r}\right), s, \varepsilon\right) \\
J_{i}\left(z_{0}\left(\tau_{i}-, c_{r}\right), \varepsilon\right)
\end{array}\right]\right)(\cdot)\right\}=0
$$

Now let condition (22) not hold. In this case, since $z(t, \varepsilon) \rightarrow z_{0}\left(t, c_{0}\right)$ as $\varepsilon \rightarrow 0$ and an operator $Z(z, t, \varepsilon)$ is continuous in $\varepsilon$ and $z$ in a neighborhood of $\varepsilon=0$ and $z_{0}\left(t, c_{0}\right)$, one can find a sufficiently small $\varepsilon>0$ such that condition (22) is not satisfied. The contradiction obtained proves Theorem 3.

By analogy with [3, p. 110; 7, p. 247], the system of equations (22) is called the equations for generating amplitudes.

If the system of equations (22) has a solution, then the vector $c_{0}$ defines that generating solution $z_{0}\left(t, c_{0}\right)$ to which the solution $z(t, \varepsilon) \in D^{n} S C_{0}$ of the initial weakly nonlinear pulse boundary-value problem (if this solution exists) tends as $\varepsilon \rightarrow 0$. However, if the system of equations (22) does not have solutions, then the boundary-value problem (21) does not have solutions in the space $D^{n} S C_{0}$. Since we perform all calculations in real form, we speak of real solutions of the system of equations for generating amplitudes.

Let us find sufficient conditions for the existence of solutions of pulse boundary-value problem (21) in the case which is called a critical case of the first order. This case is characterized by the fact that the answer to the question on the existence of solutions for the initial problem is given after the analysis of a boundary-value problem used for the determination of the first approximation to the required solution.

By changing variables

$$
z(t, \varepsilon)=z_{0}\left(t, c_{0}\right)+x(t, \varepsilon)
$$

where a constant vector $c_{0} \in R^{r}$ satisfies the system of equations (22) for the generating amplitudes, in (21), we arrive at the following problem: It is necessary to find sufficient conditions for the existence and an algorithm for construction of the solution $x(t, \varepsilon) \in D^{n} S C$ of a pulse boundary-value problem

$$
\begin{gathered}
(L x)(t)=\varepsilon Z\left(z_{0}\left(t, c_{0}\right)+x(t, \varepsilon), t, \varepsilon\right), \quad t \neq \tau_{i}, \\
\left.\Delta x\right|_{t=\tau_{i}}-S_{i} x\left(\tau_{i}-\right)=\varepsilon J_{i}\left(z_{0}\left(\tau_{i}-, c_{0}\right)+x\left(\tau_{i}-, \varepsilon\right), \varepsilon\right), \\
l x=\varepsilon J\left(z_{0}\left(\cdot, c_{0}\right)+x(\cdot, \varepsilon), \varepsilon\right)
\end{gathered}
$$

vanishing for $\varepsilon=0$.

Taking into account conditions $\left(a_{2}\right)$ and $\left(a_{3}\right)$ imposed on the nonlinearities in a neighborhood of the generating solution $z_{0}\left(t, c_{0}\right)$, let us select linear parts in $x$ and terms of zero order in $\varepsilon$ from the nonlinear operators $Z, J_{i}$, and $J$. We obtain the following expansions in a neighborhood of $x \doteq 0$ and $\varepsilon=0$ :

$$
Z\left(z_{0}+x, t, \varepsilon\right)=Z_{0}\left(t, c_{0}\right)+\left(L_{1} x(\cdot, \varepsilon)\right)(t)+R(x, t, \varepsilon)
$$

where

$$
Z_{0}\left(t, c_{0}\right)=Z\left(z_{0}\left(t, c_{0}\right), t, 0\right): D^{n} \times I_{[a, b]} \rightarrow L^{n}
$$

$L_{1}: D^{n} S C \rightarrow L^{n}$ is a linear bounded operator which is the Fréchet derivative of the operator $Z\left(z_{0}\left(t, c_{0}\right)+x(t, \varepsilon)\right.$, $t, \varepsilon)$ in $z$ for $z=z_{0}\left(t, c_{0}\right)$, and

$$
R(0, t, 0)=0, \quad \partial R(0, t, 0) \backslash \partial x=0
$$




$$
J_{i}\left(z_{0}+x, \varepsilon\right)=J_{i 0}\left(\tau_{i}-, c_{0}\right)+A_{i 1} x\left(\tau_{i}-, \varepsilon\right)+R_{i}\left(x\left(\tau_{i}-, \varepsilon\right), \varepsilon\right)
$$

where

$$
\begin{gathered}
J_{i 0}\left(\tau_{i}-, c_{0}\right)=J_{i}\left(z_{0}\left(\tau_{i}-, c_{0}\right), 0\right), \\
A_{i 1}=A_{i 1}\left(c_{0}\right)=\left.\partial J(z, 0) \backslash \partial z\right|_{z=z_{0}\left(\tau_{i}-, c_{0}\right)}, \quad i=1, \ldots, p, \\
R_{i}(0,0)=0, \quad \partial R_{i}(0,0) \backslash \partial x=0
\end{gathered}
$$

and

$$
J\left(z_{0}+x, \varepsilon\right)=J_{0}\left(\cdot, c_{0}\right)+l_{1} x(\cdot, \varepsilon)+R_{0}(x(\cdot, \varepsilon), \varepsilon)
$$

where $J_{0}\left(\cdot, c_{0}\right)=J\left(z_{0}\left(\cdot, c_{0}\right), 0\right), l_{1}: D^{n} S C \rightarrow R^{m}$ is a linear bounded $m$-dimensional vector functional which is the Fréchet derivative of a vector functional $J\left(z_{0}\left(\cdot, c_{r}^{*}\right)+x(\cdot, \varepsilon), \varepsilon\right)$ for $z=z_{0}\left(t, c_{0}\right)$, and

$$
R_{0}(0,0)=0, \quad \partial R_{0}(0,0) \backslash \partial x=0
$$

By considering the nonlinearities in boundary-value problem (23) as the inhomogeneities and applying Theorem 2, we obtain the following expression for its solution $x(t, \varepsilon)$ :

$$
x(t, \varepsilon)=\bar{X}_{r}(t) c_{r}+x^{(1)}(t, \varepsilon)
$$

where an unknown vector $c_{r}=c(t, \varepsilon)$ is determined from conditions of the form (17), (18) of the existence of the solution

$$
\begin{aligned}
& \left(P_{Y}\left\{Z_{0}\left(\cdot, c_{0}\right)+L_{1}\left[\bar{X}_{r}(\cdot) c_{r}+x^{(1)}(\cdot, \varepsilon)\right]+R(x, \cdot \varepsilon)\right\}\right)(t)=0 \\
& P_{N\left(Q^{*}\right)_{d}}\left\{J\left(\cdot, c_{0}\right)+l_{1}\left[\bar{X}_{r}(\cdot) c_{r}+x^{(1)}(\cdot, \varepsilon)\right]+R_{0}(x(\cdot, \varepsilon), \varepsilon)\right. \\
& \left.+l\left(\tilde{L}^{-}\left[\begin{array}{c}
Z_{0}\left(s, c_{0}\right)+\left(L_{1}\left[\bar{X}_{r}(\cdot) c_{r}+x^{(1)}(\cdot, \varepsilon)\right](s)+R(x, s, \varepsilon)\right. \\
J_{i 0}\left(\tau_{i}-, c_{0}\right)+A_{i 1}\left[\bar{X}_{r}\left(\tau_{i}-\right) c_{r}+x^{(1)}\left(\tau_{i}-, \varepsilon\right)\right]+R_{i}\left(x\left(\tau_{i}-, \varepsilon\right)\right.
\end{array}\right]\right)(\cdot)\right\}=0
\end{aligned}
$$

An unknown vector function $x^{(1)}(t, \varepsilon)$ is determined by the relation

$$
x^{(1)}(t, \varepsilon)=\varepsilon \bar{X}(t) Q^{+} J\left(z_{0}\left(\cdot, c_{0}\right)+x(\cdot, \varepsilon), \varepsilon\right)+\varepsilon\left(\tilde{G}\left[\begin{array}{c}
Z\left(z_{0}\left(s, c_{0}\right)+x(s, \varepsilon), s, \varepsilon\right) \\
J_{i}\left(z_{0}\left(\tau_{i}-, c_{0}\right)+x\left(\tau_{i}-, \varepsilon\right), \varepsilon\right)
\end{array}\right]\right)(t)
$$

Taking into account expansions (24)-(26) and the fact that a vector constant $c_{0} \in R^{r}$ necessarily satisfies the equation for generating amplitudes (22), in order to find the solution $x(t, \varepsilon) \in D^{n} S C, x(t, 0)=0$ of weakly nonlinear pulse boundary-value problem (23), we obtain an equivalent operator system 


$$
\begin{gathered}
x(t, \varepsilon)=\bar{X}_{r}(t) c(t, \varepsilon)+x^{(1)}(t, \varepsilon), \\
\left(B_{0} c(\cdot, \varepsilon)\right)(t)=-\left[\begin{array}{c}
\left(P_{Y}\left\{L_{1} x^{(1)}(\cdot, \varepsilon)+R(x(\cdot, \varepsilon), t, \varepsilon)\right\}\right)(t) \\
\left.P_{N\left(Q^{*}\right)_{d}}\left\{\begin{array}{c}
\left(L_{1} x^{(1)}(\cdot, \varepsilon)\right)(s)+R(x, s, \varepsilon) \\
l_{1} x^{(1)}(\cdot, \varepsilon)+R_{0}(x(\cdot, \varepsilon), \varepsilon)+l\left(\tilde{L}^{-}\left[\begin{array}{c}
(1) \\
A_{i 1} x^{(1)}\left(\tau_{i}-, \varepsilon\right)+R_{i}\left(x\left(\tau_{i}-, \varepsilon\right), \varepsilon\right)
\end{array}\right]\right)
\end{array}\right]\right), \\
x^{(1)}(t, \varepsilon)=\varepsilon\left(\tilde{G}\left[\begin{array}{c}
Z_{0}\left(s, c_{0}\right)+L_{1}\left[\bar{X}_{r}(s) c+x^{(1)}(s, \varepsilon)\right]+R(x(s, \varepsilon), s, \varepsilon) \\
J_{i 0}\left(\tau_{i}-, c_{0}\right)+A_{1 i}\left[\bar{X}_{r}\left(\tau_{i}-\right) c+x^{(1)}\left(\tau_{i}-, \varepsilon\right)\right]+R_{i}\left(x\left(\tau_{i}-, \varepsilon\right), \varepsilon\right)
\end{array}\right]\right)(t) \\
+\varepsilon \bar{X}(t) Q^{+}\left\{J_{0}\left(\cdot, c_{0}\right)+l_{1}\left[\bar{X}_{r}(\cdot) c+x^{(1)}(\cdot, \varepsilon)\right]+R_{0}(x(\cdot, \varepsilon), \varepsilon)\right\},
\end{array}\right.
\end{gathered}
$$

where

$$
\left.B_{0}=\left[P_{N\left(Q^{*}\right)_{d}}\left\{l_{1} \bar{X}_{r}(\cdot)+l\left(\tilde{L}_{Y} L_{1} \bar{X}_{r}\right)\left(\begin{array}{c}
L_{1} \bar{X}_{r}(s) \\
A_{i 1} \bar{X}_{r}\left(\tau_{i}-\right)
\end{array}\right]\right)(\cdot)\right\}\right]
$$

is a linear bounded matrix operator.

The solvability of this system depends on the solvability of the second equation.

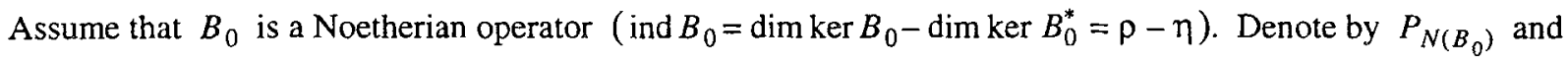
$P_{Y}$ projectors on a null space $N\left(B_{0}\right)$ and on a subspace $Y_{1}$, respectively [this subspace is isomorphic to a null space $N\left(B_{0}^{*}\right)$ of the operator $B_{0}^{*}$; let $B_{0}^{-}$denote an operator generalized inverse to the Noetherian operator $B_{0}$ $[3$, p. 53].

The second equation of operator system (27) is solvable if and only if a condition of the form (8) holds:

$$
\left.\left(P_{Y_{1}}\left[P_{N\left(Q^{*}\right)_{d}}\left\{l_{\left.P_{Y}\left\{L_{1} x^{(1)}(\cdot, \varepsilon)+R(x(\cdot, \varepsilon), t, \varepsilon)\right\}\right)(*)}+R_{0}(x(\cdot, \varepsilon), \varepsilon)+l\left(\tilde{L}^{-}\left[\begin{array}{c}
\left(L_{1} x^{(1)}(\cdot \varepsilon)\right)(s)+R(x(s, \varepsilon), s, \varepsilon) \\
\left.A_{i 1} x^{(1)}\left(\tau_{i}-, \varepsilon\right)\right]+R_{i}\left(x\left(\tau_{i}-, \varepsilon\right), \varepsilon\right)
\end{array}\right]\right)\right\}(\cdot)\right\}\right]\right)=0 .
$$

If

$$
P_{Y_{1}}\left[\begin{array}{c}
P_{Y} \\
P_{N\left(Q^{*}\right)_{d}}
\end{array}\right]=0
$$

then condition (28) always holds and the second equation of system (27) has a solution which can be represented in the form

$$
c(t, \varepsilon)=c_{\rho}^{(1)}(t)-B_{0}^{-}\left(\left[\begin{array}{c}
\left(P_{Y}\left\{L_{1} x^{(1)}(\cdot, \varepsilon)+R(x(\cdot, \varepsilon), \varepsilon)\right\}\right)(*) \\
\left.\left.P_{N\left(Q^{*}\right)_{d}}\left\{l_{1} x^{(1)}(\cdot, \varepsilon)+R_{0}(x(\cdot, \varepsilon), \varepsilon)+l\left(\tilde{L}^{-}\left[\begin{array}{c}
\left(L_{1} x^{(1)}(\cdot \varepsilon)\right)(s)+R(x(s, \varepsilon), s, \varepsilon) \\
\cdot\left[A_{i 1} x^{(1)}\left(\tau_{i}-, \varepsilon\right)\right]+R_{i}\left(x\left(\tau_{i}-, \varepsilon\right), \varepsilon\right)
\end{array}\right]\right)(\cdot)\right\}\right]\right)
\end{array}\right)(t),\right.
$$


where $c_{\rho}^{(1)}(t)=P_{N\left(B_{0}\right)_{\rho}} c(t) \in N\left(B_{0}\right)$, and $P_{N\left(B_{0}\right)_{\rho}}$ is a matrix operator formed by $\rho$ linearly independent columns of the matrix operator $P_{N\left(B_{0}\right)}$.

With regard for (29), we can rewrite operator system (27) in the form

$$
\begin{gathered}
x(t, \varepsilon)=\bar{X}_{r}(t) c(t, \varepsilon)+x^{(1)}(t, \varepsilon) \\
c(t, \varepsilon)=c_{\rho}^{(1)}(t)-\left(B_{0}^{-}\left[\begin{array}{c}
\left(P_{Y}\left\{L_{1} x^{(1)}(\cdot, \varepsilon)+R(x(\cdot, \varepsilon), \varepsilon)\right\}\right)(*) \\
\left.\left.P_{N\left(Q^{*}\right)_{d}}\left\{l_{1} x^{(1)}(\cdot, \varepsilon)+R_{0}(x(\cdot, \varepsilon), \varepsilon)+l\left(\tilde{L}^{-}\left[\begin{array}{c}
\left.\left(L_{1} x^{(1)}(\cdot, \varepsilon), \cdot \varepsilon\right)(s)+R(x(s, \varepsilon), s, \varepsilon)\right] \\
\left.A_{i 1} x^{(1)}\left(\tau_{i}-, \varepsilon\right)\right]+R_{i}\left(x\left(\tau_{i}-, \varepsilon\right), \varepsilon\right)
\end{array}\right]\right)(\cdot)\right\}\right)\right]
\end{array}\right)\right. \\
x^{(1)}(t, \varepsilon)=\varepsilon\left(\tilde{G}\left[\begin{array}{c}
Z\left(s, c_{0}\right)+L_{1}\left[\bar{X}_{r}(s) c+x^{(1)}(s, \varepsilon)\right]+R(x(s, \varepsilon), s, \varepsilon) \\
\left.J_{i 0}\left(\tau_{i}-, c_{0}\right)+A_{1 i}\left[\bar{X}_{r}\left(\tau_{i}-\right) c+x^{(1)}\left(\tau_{i}-, \varepsilon\right)\right]+R_{i}\left(x\left(\tau_{i}-, \varepsilon\right), \varepsilon\right)\right]
\end{array}\right)(t)\right. \\
+\varepsilon \bar{X}(t) Q^{+}\left\{J_{0}\left(\cdot, c_{0}\right)+l_{1}\left[\bar{X}_{r}(\cdot) c+x^{(1)}(\cdot, \varepsilon)\right]+R_{0}(x(\cdot, \varepsilon), \varepsilon)\right\}
\end{gathered}
$$

Operator system (30) belongs to a class of systems which can be solved by the method of simple iterations [7].

We construct an iterative process for finding a solution $x(t, \varepsilon) \in D^{n} S C, x(t, 0)=0$ of boundary-value problem (23) in the following way. We seek the approximations $x_{k+1}^{(1)}(t, \varepsilon)$ of $x^{(1)}(t, \varepsilon)$ as solutions of boundaryvalue problems

$$
\begin{gathered}
\left(L x_{k+1}\right)(t)=\varepsilon\left\{Z_{0}\left(t, c_{0}\right)+\left(L_{1}\left[\bar{X}(\cdot) c_{k}+x_{k}^{(1)}(\cdot, \varepsilon)\right]\right)(t)+R\left(x_{k}(t, \varepsilon), t, \varepsilon\right)\right\}, \quad t \neq \tau_{i}, \\
\left.\Delta x_{k+1}\right|_{t=\tau_{i}}-S_{i} x_{k+1}\left(\tau_{i}-, \varepsilon\right)=\varepsilon J_{i}\left(\tau_{i}-, c_{0}\right)+\varepsilon\left[A_{i 1}\left[\bar{X}\left(\tau_{i}-\right) c_{k}+x_{k}^{(1)}\left(\tau_{i}-, \varepsilon\right)\right]+R_{i}\left(x_{k}\left(\tau_{i}-, \varepsilon\right), \varepsilon\right)\right], \\
l x_{k+1}(\cdot, \varepsilon)=\varepsilon\left[J_{0}\left(\cdot, c_{0}\right)+l_{1}\left[\bar{X}(\cdot) c_{k}+x_{k}^{(1)}(\cdot \varepsilon)\right]+R_{0}\left(x_{k}(\cdot, \varepsilon), \varepsilon\right)\right] .
\end{gathered}
$$

By Theorem 2 and expansions (24)-(26), we find the solution $x_{k+1}^{(1)}(t, \varepsilon)$ of this boundary-value problem according to the relation

$$
\begin{aligned}
& x_{k+1}^{(1)}(t, \varepsilon)=\varepsilon\left(\tilde{G}\left[\begin{array}{c}
Z\left(s, c_{0}\right)+L_{1}\left[\bar{X}_{r}(s) c_{k}+x_{k}^{(1)}(s, \varepsilon)\right]+R\left(x_{k}(s, \varepsilon), s, \varepsilon\right) \\
J_{i 0}\left(\tau_{i}-, c_{0}\right)+A_{1 i}\left[\bar{X}_{r}\left(\tau_{i}-\right) c_{k}+x_{k}^{(1)}\left(\tau_{i}-, \varepsilon\right)\right]+R_{i}\left(x_{k}\left(\tau_{i}-, \varepsilon\right), \varepsilon\right)
\end{array}\right]\right)(t) \\
& +\varepsilon \bar{X}(t) Q^{+}\left\{J_{0}\left(\cdot, c_{0}\right)+l_{1}\left\{\bar{X}_{r}(\cdot) c_{k}+x_{k}^{(1)}(\cdot, \varepsilon)\right\}+R_{0}\left(x_{k}(\cdot, \varepsilon), \varepsilon\right)\right\}
\end{aligned}
$$

The necessary and sufficient condition of the solvability of this boundary-value problem leads to an operator equation

$$
\left.\left(B_{0} c_{k}(\cdot, \varepsilon)\right)(t)=-\left[P_{N\left(Q^{*}\right)_{d}}\left\{l_{1} x_{k}^{(1)}(\cdot, \varepsilon)+R_{0}\left(x_{k}(\cdot \varepsilon), \varepsilon\right)+l\left[\tilde{L}^{-}\left[\begin{array}{c}
\left(L_{1} x_{k}^{(1)}(\cdot, \varepsilon)\right)(s)+R(x(s, \varepsilon), s, \varepsilon) \\
\left.A_{i 1} x_{k}^{(1)}\left(\tau_{i}-, \varepsilon\right)\right]+R_{i}\left(x\left(\tau_{i}-, \varepsilon\right), \varepsilon\right)
\end{array}\right]\right)\right](\cdot)\right\}\right]
$$


from which we determine a $k$ th approximation $c_{k}(t, \varepsilon)$ of $c(t, \varepsilon)$. Solvability criteria for systems of the form (31) hold on every step of the iterative process if

$$
P_{Y_{1}}\left[\begin{array}{c}
P_{Y} \\
P_{N\left(Q^{*}\right)_{d}}
\end{array}\right]=0
$$

The approximation $x_{k+1}(t, \varepsilon)$ of $x(t, \varepsilon)$ has the form

$$
x_{k+1}(t, \varepsilon)=\bar{X}_{r}(t) c_{k}+x_{k+1}^{(1)}(t, \varepsilon)
$$

Thus, the following theorem is true:

Theorem 4 (sufficient condition). Let boundary-value problem (21) satisfy conditions $\left(a_{1}\right)-\left(a_{5}\right)$ and let generating boundary-value problem (4)-(6) satisfy the conditions of Theorem 2. Then, for every value of the vector $c_{0} \in R^{r}$ satisfying the system of equations (22) for generating amplitudes, boundary-value problem (21) has a $\rho$-parametric family of solutions $z(t, \varepsilon) \in D^{n} S C_{0}$ if the condition

$$
P_{Y_{1}}\left[\begin{array}{c}
P_{Y} \\
P_{N\left(Q^{*}\right)_{d}}
\end{array}\right]=0
$$

is satisfied. These solutions can be determined by the following iterative process convergent on $\left[0, \varepsilon_{*}\right] \subseteq\left[0, \varepsilon_{0}\right]$ :

$$
\begin{gathered}
z_{k+1}(t, \varepsilon)=z_{0}\left(t, c_{0}\right)=x_{k+1}(t, \varepsilon), \\
x_{k+1}(t, \varepsilon)=\bar{X}_{\rho}(t) c_{\rho}^{(1)}(t)+\bar{X}_{r}(t) c_{k}(t, \varepsilon)+x_{k+1}^{(1)}(t, \varepsilon), \\
c(t, \varepsilon)=c_{\rho}^{(1)}(t)-\left(B_{0}^{-}\left[P_{Y}\left\{L_{1} x_{k}^{(1)}(\cdot, \varepsilon)+R\left(x_{k}(\cdot, \varepsilon), \varepsilon\right)\right\}\right)(\cdot)\right. \\
{\left[P _ { N ( Q ^ { * } ) _ { d } } \left\{\begin{array}{c}
\left.\left.\left.l_{1} x_{k}^{(1)}(\cdot, \varepsilon)+R_{0}(x(\cdot, \varepsilon), \varepsilon)+l\left[\tilde{L}^{-}\left[\begin{array}{l}
\left(L_{1} x_{k}^{(1)}(\cdot, \varepsilon)\right)(s)+R\left(x_{k}(s, \varepsilon), s, \varepsilon\right) \\
\left.A_{i 1} x_{k}^{(1)}\left(\tau_{i}-, \varepsilon\right)\right]+R_{i}\left(x_{k}\left(\tau_{i}-, \varepsilon\right), \varepsilon\right)
\end{array}\right]\right)(\cdot)\right\}\right]\right)(t), \\
x_{k+1}^{(1)}(t, \varepsilon)=\varepsilon\left(\tilde{G}\left[\begin{array}{c}
Z_{0}\left(s, c_{0}\right)+L_{1}\left[\bar{X}_{r}(s) c_{k}+x_{k}^{(1)}(s, \varepsilon)\right]+R\left(x_{k}(s, \varepsilon), s, \varepsilon\right) \\
J_{i 0}\left(\tau_{i}-, c_{0}\right)+A_{1 i}\left[\bar{X}_{r}\left(\tau_{i}-\right) c_{k}+x_{k}^{(1)}\left(\tau_{i}-, \varepsilon\right)\right]+R_{i}\left(x_{k}\left(\tau_{i}-, \varepsilon\right), \varepsilon\right)
\end{array}\right]\right)(t) \\
+\varepsilon \bar{X}(t) Q^{+}\left\{J_{0}\left(\cdot, c_{0}\right)+l_{1}\left[\bar{X}_{r}(\cdot) c_{k}+x_{k}^{(1)}(\cdot, \varepsilon)\right]+R_{0}(x(\cdot, \varepsilon), \varepsilon)\right\} .
\end{array}\right.\right.}
\end{gathered}
$$

The interval $\left[0, \varepsilon_{*}\right] \subseteq\left[0, \varepsilon_{0}\right]$ on which iterative process (33) is convergent can be determined by the majorizing Lyapunov equations [8] similarly to the case of boundary-value problems for ordinary differential systems $[3,7]$.

Remark 3. If the linear operator $B_{0}$ is a Noetherian operator and $\operatorname{dim} \operatorname{ker} N\left(B_{0}\right)=0$, then the weakly nonlinear boundary-value problem (21) has a unique solution $z(t, \varepsilon) \in D^{n} S C_{0}$ that is transformed into the 
generating solution $z\left(t, c_{0}\right)$ with a constant $c_{0}$ satisfying the system of equations (22) for generating amplitudes.

Remark 4. If the linear operator $B_{0}$ is a Fredholm one and $\operatorname{dim} \operatorname{ker} N\left(B_{0}\right)=0$, then, due to the fact that $P_{Y_{1}}=0$ and $P_{N\left(B_{0}\right)}=0$, condition (32) holds automatically. In this case, the weakly nonlinear pulse boundaryvalue problem (21) also has a unique solution and, in iterative procedure (33), the generalized inverse operator $B_{0}^{-}$ is replaced by inverse operator $B_{0}^{-1}$.

Remark 5. If initial operator equation (4) is everywhere solvable ( $\operatorname{dim} \operatorname{ker} L^{*}=0$ ), then a projector $P_{Y}$ is equal to zero and condition (32) takes the form $P_{Y_{1}} P_{N\left(Q^{*}\right)_{d}}=0$.

As an example of a boundary-value problem of this sort, we consider a weakly nonlinear boundary-value problem for a pulse differential system with lag.

\section{Weakly Nonlinear Boundary-Value Problems for Pulse Differential Systems with Lag}

Preserving the notation of [4], we consider a weakly nonlinear boundary-value problem for pulse differential systern with lag

$$
\begin{gathered}
(L z)(t) \equiv \dot{z}(t)-A(t)\left(S_{h} z\right)(t)=f(t)+\varepsilon Z\left(\left(S_{h} z\right), t, \varepsilon\right), \\
\left.\Delta\right|_{t=\tau_{j}}-B_{j} z\left(\tau_{i}-\right)=a_{j}+\varepsilon J_{j}\left(\left(S_{h^{2}} z\right)\left(\tau_{j}-, \varepsilon\right), \varepsilon\right) \\
l z=\alpha+\varepsilon J\left(\left(S_{h^{2}} z\right)(\cdot, \varepsilon), \varepsilon\right)
\end{gathered}
$$

under the following assumptions:

$\left(b_{1}\right)$ the columns of an $(n \times N)$-dimensional matrix $A(t)$ and an $n$-dimensional vector $f(t)$ belong to the space $L^{n}$;

$\left(\mathrm{b}_{2}\right) \quad S_{h}: D^{n} \rightarrow L^{N}$ is an inner substitution operator defined by the equality $S_{h}=\operatorname{col}\left[S_{h_{1}}, S_{h_{2}}, \ldots, S_{h_{k}}\right]$, where $h_{i}(t)$ are measurable on $[a, b], h_{i}(t) \leq t, i=1,2, \ldots, k$, and $N=n k$;

$\left(b_{3}\right)$ a nonlinear $n$-dimensional vector function $Z(y, t, \varepsilon)$ is continuously differentiable in $y$, continuous in $\varepsilon$, and summable in $t$ in a neighborhood of $\varepsilon=0$ and of the solutions $z\left(t, c_{0}\right)$ of the generating boundary-value problem;

$\left(b_{4}\right)$ nonlinear $n$ - and $m$-dimensional functionals $J_{j}\left(y\left(\tau_{j}-, \varepsilon\right), \varepsilon\right)$ and $J(y(\cdot, \varepsilon), \varepsilon)$ are (Fréchet) continuously differentiable with respect to the first argument in a neighborhood of $z\left(t, c_{0}\right)$ and $\varepsilon=0$, and are continuous in $\varepsilon$ for $\varepsilon \in\left[0, \varepsilon_{0}\right]$;

$\left(b_{5}\right) \quad l$ is a linear bounded $m$-dimensional vector functional, $l: D^{n} S \rightarrow R^{n}$, and $a_{j} \in R^{n}, j=1,2, \ldots, p$;

(b) $\alpha \in R^{m}$.

We consider the problem of finding existence conditions and an algorithm for construction of the solution $z(t$, 
$\varepsilon$ ) which belongs to the space $D^{n} S$ with respect to the first argument, belongs to the space of continuous functions $C\left[0, \varepsilon_{0}\right]$ with respect to the second argument, and, for $\varepsilon=0$, is reduced to one of the generating solutions $z_{0}\left(t, c_{r}\right)$ of the generating boundary-value problem that is obtained from (34) if $\varepsilon=0$.

Assume that $f(t) \in L^{n}, \alpha \in R^{m}$, and $a_{j} \in R^{n}$ satisfy the conditions of Theorem 2. Due to the everywhere solvability of the Cauchy problem for the system with lag [condition (17) always holds], these conditions take the form [4]

$$
P_{N\left(Q^{*}\right)_{d}}\left\{\alpha-l \int_{a}^{b} \bar{K}(\cdot, s) f(s) d s-\sum_{i=1}^{p} l \bar{X}_{i}(\cdot) a_{i}\right\}=0
$$

where

$$
\begin{gathered}
\bar{K}_{i}(t, s)=X(t) \prod_{v=k}^{i+1} X^{-1}\left(\tau_{v}\right)\left(E+B_{v}\right) X\left(\tau_{v}\right) X^{-1}\left(\tau_{i}\right) B_{i} K\left(\tau_{i}, \varepsilon\right), \\
\bar{X}_{i}(t)=X(t) \prod_{v=k}^{i+1} X^{-1}\left(\tau_{v}\right)\left(E+B_{v}\right) X\left(\tau_{v}\right) X^{-1}\left(\tau_{i}\right), \\
a<\tau_{k-1}<s<\tau_{i}<\tau_{k}<t \leq \tau_{k+1}<b .
\end{gathered}
$$

Here,

$$
\prod_{v=k}^{i+1} X^{-1}\left(\tau_{v}\right)\left(E+B_{v}\right) X\left(\tau_{v}\right)=E
$$

if $k>i+1$ and $K(t, s)$ is a Cauchy matrix for the differential system with lag. In this case, the generating boundary-value problem for (34) has an $r$-parametric family of solutions, which, for the pulse systems with lag, can be written in the form [4]

$$
z\left(t, c_{r}\right)=\bar{X}_{r}(t) c_{r}+\left(G\left[\begin{array}{c}
f(\cdot) \\
a_{j}
\end{array}\right]\right)(t)+\bar{X}(t) Q^{+} \alpha
$$

where $\bar{X}_{r}(t)=\bar{X}_{r}(t) P_{N(Q)}$ is an $(n \times r)$-dimensional fundamental matrix of the homogeneous pulse boundaryvalue problem (5), (6) and

$$
\left(G\left[\begin{array}{l}
* \\
*
\end{array}\right]\right)(t)
$$

is a generalized Green operator for semihomogeneous pulse boundary-value problem (5), (6) having the form

$$
\left(G\left[\begin{array}{c}
f(\cdot) \\
a_{j}
\end{array}\right]\right)(t)=\int_{a}^{b} K(t, s) f(s) d s-\bar{X}(t) Q^{+} l \int_{a}^{b} K(\cdot, s) f(s) d s
$$




$$
\begin{aligned}
& +\sum_{i=1}^{k} \int_{a}^{\tau_{i}} \bar{K}_{i}(t, s) f(s) d s-\bar{X}(t) Q^{+} \sum_{i=1}^{p} l \int_{a}^{\tau_{i}} \bar{K}_{i}(\cdot, s) f(s) d s \\
& +\sum_{i=1}^{k} \bar{X}_{i}(t) a_{i}-\bar{X}(t) Q^{+} \sum_{i=1}^{p} l \bar{X}_{i}(\cdot) a_{i}+\bar{X}(t) Q^{+} \alpha .
\end{aligned}
$$

We now consider necessary and sufficient conditions for the existence of solutions of boundary-value problem (34).

Theorem 5 (necessary condition). Let the weakly nonlinear boundary-value problem (34) with pulse influences at fixed times have a solution $z(t, \varepsilon) \in D^{n} S C$. Assume that, for $\varepsilon=0$, this solution is reduced to one of the generating solutions $z_{0}\left(t, c_{r}\right)$ of the linear Noetherian boundary-value problem with a constant $c_{r}=c_{0} \in R^{r}$ which is generating for problem (34). Then the vector $c_{0}$ satisfies the equation for generating amplitudes of the form (22):

$$
P_{N\left(Q^{*}\right)_{d}}\left\{J\left(\left(S_{h} z_{0}\right)\left(\cdot, c_{r}\right), 0\right)-l \int_{a}^{b} \bar{K}_{i}(\cdot, s) Z\left(\left(S_{h} z_{0}\right)\left(, c_{r}\right)(s), s, 0\right) d s-\sum_{i=1}^{p} l \bar{X}_{i}(\cdot) J_{j}\left(\left(S_{h} z_{0}\right)\left(\tau_{j}, c_{r}\right), 0\right)\right\}=0
$$

where $P_{N\left(Q^{*}\right)_{d}}$ is a $(d \times m)$ matrix whose rows are $d$-linearly independent rows of the matrix $P_{N\left(Q^{*}\right)}: R^{m} \rightarrow$ $N\left(Q^{*}\right), Q=l X(\cdot)$, and $X(t)$ is a fundamental matrix of the pulse differential system with lag.

Note that, due to the everywhere solvability of the initial pulse differential system with lag, system (22) consists of a single equation.

In order to find sufficient conditions for the existence of a solution of boundary-value problem (34) and an algorithm of its construction, we reduce this problem in the way already described to an equivalent operator system. By analyzing this system, we obtain the sufficient condition for the existence of solution $z(t, \varepsilon)$ of the initial boundary-value problem.

After changing the variables $z(t, \varepsilon)=z_{0}\left(t, c_{0}\right)+x(t, \varepsilon)$, where $z_{0}\left(t, c_{0}\right)$ is a generating solution with a vector constant $c_{0} \in R^{r}$, which satisfies the equation for generating amplitudes (35), we obtain the boundary-value problem

$$
\begin{gathered}
(L x)(t) \equiv \dot{x}(t)-A(t) S_{h} x(t)+Z\left(\left(S_{h}\left[z_{0}\left(\cdot, c_{0}\right)+x(\cdot, \varepsilon)\right]\right), t, \varepsilon\right), \quad t \neq \tau_{i} \\
\left.\Delta\right|_{t=\tau_{j}}-B_{j} x\left(\tau_{i}-\right)=\varepsilon J_{j}\left(S_{h}\left[z_{0}\left(\cdot, c_{0}\right)+x(\cdot, \varepsilon)\right]\left(\tau_{j}-\right), \varepsilon\right) \\
l x(\cdot)=\varepsilon J\left(S_{h}\left[z_{0}\left(\cdot, c_{0}\right)+x(\cdot, \varepsilon)\right], \varepsilon\right)
\end{gathered}
$$

for finding the deviation of $x(t, \varepsilon)$ from the generating solution, which belongs to the space $D^{n} S C$ and vanishes for $\varepsilon=0$.

By using conditions $\left(\mathrm{b}_{3}\right)$ and $\left(\mathrm{b}_{4}\right)$ on the nonlinearities $Z, J_{j}$, and $J$ in a neighborhood of the generating solution $z_{0}\left(t, c_{0}\right)$, we select from the vector functions $Z\left(S_{h}\left[z_{0}+x\right], t, \varepsilon\right), J_{j}\left(S_{h}\left[z_{0}+x\right], \varepsilon\right)$, and $J\left(S_{h}\left[z_{0}+x\right]\right.$, $\varepsilon)$ their linear parts in $x$ and terms of zero order in $\varepsilon$ :

$$
Z\left(\left(S_{h}\left[z_{0}\left(\cdot, c_{0}\right)+x(\cdot, \varepsilon]\right)(t), t, \varepsilon\right)=Z\left(\left(S_{h} z_{0}\left(\cdot, c_{0}\right)\right)(t), t, 0\right)+A_{1}(t)\left(S_{h} x(\cdot, \varepsilon)\right)(t)+R\left(\left(S_{h} x(\cdot, \varepsilon)(t), t, \varepsilon\right),\right.\right.
$$


where

$$
A_{1}(t)=A_{1}\left(t, c_{0}\right)=\left.\frac{\partial Z\left(S_{h} z, t, 0\right)}{\partial S_{h} z}\right|_{\left(S_{h} z\right)(t)=\left(S_{h} z_{0}\right)\left(t, c_{0}\right)}
$$

is an $(n \times N)$-matrix of the class $L^{n}$,

$$
\begin{gathered}
R(0, t, 0)=0, \quad \partial R(0, t, 0) / \partial S_{h} x=0 ; \\
\left.\left.J_{j}\left(S_{h}\left[z_{0}\left(\cdot, c_{0}\right)+x(\cdot, \varepsilon)\right]\right)\left(\tau_{j}-\right), \varepsilon\right)=J_{j}\left(S_{h} z_{0}\left(\cdot, c_{0}\right)\right)\left(\tau_{j}-\right), 0\right) \\
+A_{1 j}\left(S_{h} x(\cdot, \varepsilon)\right)\left(\tau_{j}-\right)+R_{j}\left(S_{h}\left(x(\cdot, \varepsilon)\left(\tau_{j}-\right), \varepsilon\right),\right.
\end{gathered}
$$

where

$$
A_{1 j}=\left.\frac{\partial J_{j}\left(S_{h} z, 0\right)}{\partial S_{h} z}\right|_{S_{h} z=S_{h} z_{0}\left(\tau_{j}-, c_{0}\right)}, \quad j=1, \ldots, p
$$

are $(n \times N)$-dimensional constant matrices,

$$
\begin{gathered}
R_{j}(0,0)=0, \quad \partial R_{j}(0,0) / \partial S_{h} x=0, \\
J\left(S_{h}\left[z_{0}\left(\cdot, c_{0}+x(\cdot, \varepsilon)\right](\cdot), \varepsilon\right)=J\left(S_{h} z_{0}\left(\cdot, c_{0}\right)\right)(\cdot), 0\right)+l_{1}\left(S_{h} x(\cdot, \varepsilon)\right)(\cdot)+R_{0}\left(S_{h} x(\cdot, \varepsilon)(\cdot), \varepsilon\right),
\end{gathered}
$$

$l_{1}$ is a linear part of a vector functional

$$
\begin{gathered}
J\left(z_{0}\left(\cdot, c_{0}\right)+x(\cdot \varepsilon), \varepsilon\right) \\
R_{0}(0,0)=0, \quad \frac{\partial R_{0}(0,0)}{\partial S_{h} x}=0
\end{gathered}
$$

Theorem 6 (sufficient condition). Let boundary-value problem (34) satisfy conditions $\left(b_{1}\right)-\left(b_{6}\right)$ and let the generating boundary-value problem satisfy the conditions of Theorem 2. Then, for every value of the vector $c_{0} \in R^{r}$ satisfying the equation for generating amplitudes (35), under the condition

$$
P_{N\left(B_{0}^{*}\right)} P_{N\left(Q^{*}\right)_{d}}=0
$$

boundary-value problem (34) has a $\rho$-parametric family of solutions $z(t, \varepsilon) \in D^{n} S C$, which is reduced to a generating one $z_{0}\left(t, c_{0}\right)$ for $\varepsilon=0$. These solutions can be determined by means of the following iterative process convergent on $\left[0, \varepsilon_{*}\right] \subseteq\left[0, \varepsilon_{0}\right]$ :

$$
\begin{gathered}
z_{k+1}(t, \varepsilon)=z_{0}\left(t, c_{0}\right)+x_{k+1}(t, \varepsilon), \\
x_{k+1}(t, \varepsilon)=\bar{X}_{\rho}(t) c_{\rho}+\bar{X}_{r}(t) c_{k}^{(1)}+x_{k+1}^{(1)}(t, \varepsilon),
\end{gathered}
$$




$$
\begin{aligned}
& c_{k}=P_{N\left(B_{0}\right)_{\rho}} c_{\rho}+c_{k}^{(1)}=P_{N\left(B_{0}\right)_{\rho}} c_{\rho}+B_{0}^{+} P_{N\left(Q^{*}\right)_{d}}\left\{l_{1} S_{h} x_{k}^{(1)}(\cdot \varepsilon)+R_{0}\left(S_{h} x_{k}(\cdot, \varepsilon), \varepsilon\right)\right. \\
& -l \int_{a}^{b} \widetilde{K}(\cdot, s)\left[A_{1}(s)\left(S_{h} x_{k}^{(1)}(, \varepsilon)\right)(s)+R\left(S_{h} x_{k}(\cdot, \varepsilon)\right)(s), s, \varepsilon\right] d s \\
& -\sum_{i=1}^{p}\left\{\bar{X}_{i}(\cdot)\left[A_{1 i}(s)\left(S_{h} x^{(1)}(\cdot, \varepsilon)\right)\left(\tau_{i}-\right)+R_{i}\left(S_{h} x_{k}(\cdot, \varepsilon)\left(\tau_{i}-\right), s, \varepsilon\right)\right]\right\} \\
& x_{k+1}^{(1)}(t, \varepsilon)=\varepsilon \bar{X}(t) Q^{+}\left\{J\left(S_{h} z_{0}\left(\cdot, c_{0}\right), 0\right)+l_{1} S_{h}\left[X_{r}(\cdot) c_{k}+x_{k}^{(1)}(\cdot, \varepsilon)\right]+R_{0}\left(S_{h} x_{k}(\cdot, \varepsilon)\right)\right\} \\
& +\varepsilon\left(G\left[\begin{array}{c}
Z\left(\left(S_{h} z_{0}\left(\cdot, c_{0}\right)(*)\right), *, 0\right)+A_{1}(*) S_{h}\left[\bar{X}_{r}(\cdot) c_{k}+x_{k}^{(1)}(\cdot, \varepsilon)\right](*)+R\left(\left(S_{h} x_{k}(\cdot, \varepsilon)(*), *, \varepsilon\right)\right. \\
J_{j}\left(\left(S_{h} z_{0}\left(\cdot, c_{0}\right)\right)\left(\tau_{j}-\right), 0\right)+A_{1 j} S_{h}\left[\bar{X}_{r}(\cdot) c_{k}+x_{k}^{(1)}(\cdot, \varepsilon)\right]\left(\tau_{j}-\right)+R_{j}\left(S_{h} x_{k}(\cdot, \varepsilon)\left(\tau_{j}-\right), \varepsilon\right)
\end{array}\right]\right)(t), \\
& x_{0}(t, \varepsilon)=x_{0}^{(1)}(t, \varepsilon)=0,
\end{aligned}
$$

where

$$
B_{0}=P_{N\left(Q^{*}\right)_{d}}\left\{l_{1} S_{h} \bar{X}_{r}(\cdot)-l \int_{a}^{b} \bar{K}(\cdot, s) A_{1}(s) S_{h} \bar{X}_{r}(\cdot) d s-\sum_{i=1}^{p} l \bar{X}_{i}(\cdot) A_{l i}(s)\left(S_{h} \bar{X}_{r}\right)\left(\tau_{i}\right)\right\}
$$

is a $(d \times m)$-dimensional constant matrix.

Remark 6. In the case where $P_{N\left(B_{0}\right)}=0$, the weakly nonlinear pulse problem (34) has a unique solution $z(t, \varepsilon) \in D^{n} S C$ which is reduced to a generating one for $\varepsilon=0$.

Remark 7. In the case of Fredholm boundary-value problems $(m=n)$, the condition $P_{N\left(B_{0}\right)}=0$ implies that $P_{N\left(B_{0}^{*}\right)}=0$, whence condition $P_{N\left(B_{0}^{*}\right)} P_{N\left(Q^{*}\right)_{d}}=0$ holds automatically. It follows from $P_{N\left(B_{0}\right)}=0$ that $\operatorname{det} B_{0} \neq 0$, and $B_{0}^{+}$is replaced by $B_{0}^{-1}$ in iterative procedure (36).

\section{REFERENCES}

1. A. M. Samoilenko and N. A. Perestyuk, Differential Equations with Pulse Influence, [in Russian], Vyshcha Shkola, Kiev (1987).

2. A. Halanay and D. Wexler, Qualitative Theory of Pulse Systems [In Romanian], Editura Academiei Rep. Soc. Romania, Bucharest (1968).

3. A. A. Boichuk, V. F. Zhuravlev, and A. M. Samoilenko, Generalized Inverse Operators and Noether Boundary-Value Problems [in Russian], Institute of Mathematics, Ukrainian Academy of Sciences, Kiev (1995).

4. A. A. Boichuk, V. F. Zhuravlev, and A. M. Samoilenko, "Linear Noether boundary-value problems for pulse differential systems with lag," Differents. Uravn., 30, No. 10, 1677-1682 (1994).

5. S. G. Krein, Linear Equations in Banach Space [in Russian], Nauka, Moscow (1971).

6. N. V. Azbelev, V. P. Maksimov, and L. F. Rakhmatullina, Introduction to the Theory of Functional Differential Equations [in Russian], Nauka, Moscow (1991).

7. E. A. Grebenikov and Yu. A. Ryabov, Constructive Methods of Analysis of Nonlinear Systems [in Russian], Nauka, Moscow (1979).

8. L. V. Kantorovich, "Principle of the Lyapunov majorants and the Newton method," Dokl. Akad Nauk SSSR, 76, No. 1, 17-20 (1951). 